\title{
Explaining the XENON1T Excess with Luminous Dark Matter
}

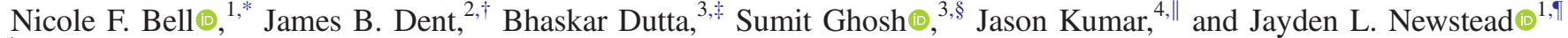 \\ ${ }^{1}$ ARC Centre of Excellence for Dark Matter Particle Physics, School of Physics, The University of Melbourne, Victoria 3010, Australia \\ ${ }^{2}$ Department of Physics, Sam Houston State University, Huntsville, Texas 77341, USA \\ ${ }^{3}$ Mitchell Institute for Fundamental Physics and Astronomy, Department of Physics and Astronomy, Texas A\&M University, \\ College Station, Texas 77843, USA \\ ${ }^{4}$ Department of Physics, University of Hawaii, Honolulu, Hawaii 96822, USA
}

(Received 25 June 2020; accepted 9 September 2020; published 12 October 2020)

\begin{abstract}
We show that the excess in electron recoil events seen by the XENON1T experiment can be explained by a relatively low-mass luminous dark matter candidate. The dark matter scatters inelastically in the detector (or the surrounding rock) to produce a heavier dark state with a $\sim 2-3 \mathrm{keV}$ mass splitting. This heavier state then decays within the detector, producing a peak in the electron recoil spectrum that is a good fit to the observed excess. We comment on the ability of future direct detection experiments to differentiate this model from other "beyond the standard model" scenarios and from possible tritium backgrounds, including the use of diurnal modulation, multichannel signals, etc., as possible distinguishing features of this scenario.
\end{abstract}

DOI: 10.1103/PhysRevLett.125.161803

Introduction.-Recently the XENON Collaboration announced an excess of low-energy electron recoil events above their expected background [1]. Though this excess may originate from a tritium $\beta$ decay that was previously not included in their background model, the collaboration also examined "beyond the standard model" (BSM) possibilities that included solar axions and a neutrino magnetic moment $\left(\mu_{\nu}\right)$ [2-4]. With a trace amount of tritium $\left(6.2 \pm 2.0 \times 10^{-20} \mathrm{~mol} / \mathrm{mol}\right)$ added to the background model, the anomaly is explained at $3.2 \sigma$ significance, while the background plus solar axion (background plus $\mu_{\nu}$ ) solution provides a $3.5 \sigma(3.2 \sigma)$ significance fit to the excess within certain parameter ranges. These BSM possibilities lose substantial statistical significance when combined with a tritium component in the fit-down to $2.1 \sigma(0.9 \sigma)$ for the solar axion $\left(\mu_{\nu}\right)$ case. It should also be noted that the axion explanation of the excess is in conflict with the astrophysical constraints [5]. Additionally, the collaboration examined the possibility of bosonic dark matter but found no global significance above $3 \sigma$. Other studies of BSM explanations for the excess include [6-21].

The XENON1T excess is characterized by a peak at $\sim 3 \mathrm{keV}$. In this Letter, we consider the possibility that the XENON1T excess is generated by the interactions of luminous dark matter (LDM) [22-24] with a mass splitting in the

Published by the American Physical Society under the terms of the Creative Commons Attribution 4.0 International license. Further distribution of this work must maintain attribution to the author(s) and the published article's title, journal citation, and DOI. Funded by SCOAP ${ }^{3}$. $\delta \sim 3 \mathrm{keV}$ range. The basic idea is that dark matter scattering is purely inelastic, with the dark matter $(\chi)$ scattering off nuclei (either in the detector or in the surrounding overburden) to produce an excited dark state $\left(\chi^{\prime}\right)$. The dark state then decays $\left(\chi^{\prime} \rightarrow \chi \gamma\right)$ by the emission of a monoenergetic photon with energy $\sim \delta$. Given the energy resolution of XENON1T, the resulting electron recoil spectrum contains a peak that is a good fit to the XENON1T excess.

The Letter is organized as follows. First, we briefly review the setup of luminous dark matter and its application to the XENON1T excess. Next, we present our results. Following that, we discuss the prospects for future experiments to probe this model. We then conclude with a discussion of our results.

Luminous dark matter.-Our basic model is a species of luminous dark matter. This is a two-state inelastic dark matter scenario in which the heavier dark state produces photons via its decays. Specifically, the cosmological cold dark matter is a particle $\chi$ with mass $m_{\chi}$, and there exists a slightly heavier dark state $\chi^{\prime}$, whose mass exceeds $m_{\chi}$ by the mass splitting $\delta=m_{\chi^{\prime}}-m_{\chi} \ll m_{\chi}$. The dominant decay of $\chi^{\prime}$ is through $\chi^{\prime} \rightarrow \chi \gamma$. Indeed, if $\delta$ is sufficiently small and if $\chi^{\prime}$ and $\chi$ have the same spin, this is the only visible decay that will be accessible (a two neutrino final state would also be possible). Note that, if $\delta \ll m_{\gamma}$, then in the rest frame of the $\chi^{\prime}$ we will find $E_{\gamma}=\delta+\mathcal{O}\left(\delta^{2} / m_{\chi}\right)$. Note that even if $\chi^{\prime}$ decays to $\chi$ and multiple photons, the sum of photon energies will be $\delta+\mathcal{O}\left(\delta^{2} / m_{\chi}\right)$ because the outgoing $\chi$ will have negligible kinetic energy for $\delta / m_{\chi} \ll 1$. This scenario can emerge if the dark matter is coupled to a mediator, $\phi$, through a $\chi \chi^{\prime} \phi$ interaction with $\phi$ decaying to $\gamma \gamma$. 
In this scenario, dark matter scattering is entirely inelastic $\left(\chi A \rightarrow \chi^{\prime} A\right)$. This type of purely inelastic scattering arises generically in a variety of contexts [23,25-40]. For example, inelastic scattering mediated by a dark photon with a vector coupling to the dark matter is generic in any model where dark matter is only charged under spontaneously broken continuous symmetries. The reason is that a gauge boson can only couple to a complex degree of freedom. But if all of the continuous symmetries under which the dark matter is charged are spontaneously broken, then the dark matter is generically expected to split into two real degrees of freedom. Since one cannot form a vector current with a single real degree of freedom, the dark photon must instead couple to an off-diagonal vector current, yielding inelastic scattering. Moreover, a small mass splitting can be technically natural, e.g., in models where the two dark states form a pseudo-Dirac fermion.

As with the ambient dark matter particles, $\chi$, the $\chi^{\prime}$ produced from inelastic scattering is nonrelativistic. Therefore, the eventual decay of the $\chi^{\prime}$ yields nearly monoenergetic photons in the frame of the Earth. This spectrum will have a peak at $\delta$ and a width of roughly $\beta \delta$, where $\beta \sim \mathcal{O}\left(10^{-3}\right)$ is the approximate velocity of $\chi^{\prime}$ in the frame of the Earth. For our purposes, this is essentially a line signal. But this monoenergetic signal will be smeared by the energy resolution of the detector. Note also that, in order for inelastic scattering to be kinematically allowed, one must have $\delta \lesssim m_{\chi} v^{2}$; if $\delta \sim \mathcal{O}(\mathrm{keV})$, then we must have $m_{\chi} \gtrsim \mathcal{O}(\mathrm{GeV})$.

Note that if the lifetime of $\chi^{\prime}$ is short, $\mathcal{O}(1 \mu \mathrm{s})$, it will decay within the XENON1T fiducial volume if the initial inelastic scatter itself took place within this volume. In that case, it is possible for the initial scatter to also produce a detectable signal, either in the form of a nuclear recoil or an electron recoil via the Migdal effect [41-44]. However, we shall see that, for the inelastic cross section required to explain the XENON1T electron recoil excess, the corresponding nuclear recoil signal is below current experimental sensitivity when $m_{\chi} \lesssim 15 \mathrm{GeV}$. Additionally, the upscatter could cause some events to be removed due to the multiscatter veto, reducing the detection efficiency. Lastly, the Migdal process will be a subleading effect, since only a very small fraction of inelastic scatters will produce a Migdal electron, whereas every inelastic scatter will produce a photon via $\chi^{\prime}$ decay.

If the lifetime of the $\chi^{\prime}$ is longer, then it is not necessary for the initial scatter to even occur within the detector. Instead the dark matter could scatter within the surrounding rock, with the produced $\chi^{\prime}$ decaying within the detector. Provided that the decay length of the $\chi^{\prime}$ is at most comparable to the length of the overburden, one would find that the rate of $\chi^{\prime}$ decay in the detector is similar to the rate of dark matter scattering in the detector. In this case, there will be some differences between the rate of scattering and the rate of $\chi^{\prime}$ decay in the detector due to the differing densities and compositions of the detector versus the surrounding material. But this has little effect on our main result.

The xenon excess and LDM.-The excess events observed by XENON1T are tightly restricted to the energy range of $2-7 \mathrm{keV}$, with the most significant deviations within just 2 bins of $2-4 \mathrm{keV}$. Such a narrowly peaked signal can be fit with a monoenergetic photon once smearing due to the detector resolution has been taken into account. The energy resolution of the XENON1T detector can be modeled as a Gaussian with width

$$
\frac{\sigma(E)}{\mathrm{keV}}=0.31 \sqrt{\frac{E}{\mathrm{keV}}}+0.0035 \frac{E}{\mathrm{keV}}
$$

which gives a width of $\sim 18.5 \%$ at $E=2.8 \mathrm{keV}$, which is in good agreement with the calibration data $[1,45]$. The detection efficiency of low-energy electron recoils is taken from [1]. The signal model is defined by two parameters: the line position in energy and the integrated rate. We perform a two parameter fit to the first 14 bins to find the best-fit signal model by minimizing the $\chi^{2}$ between the data and the signal plus background events. Including additional bins does not affect the best-fit point but does help evaluate the relative goodness of fit of the signal models. We find that a line energy of $E_{\gamma}=2.75 \mathrm{keV}$ and rate of 69.8 events/(tonne $\times$ year) provides a good fit to the data: $\chi^{2} /$ d.o.f $=0.42$, with $\Delta \chi^{2}=11.4$, compared to the background-only model as demonstrated in Fig. 1. We also have included the best fit to the excess with LDM plus an unconstrained tritium component. The best-fit line signal shifts down slightly to $2.72 \mathrm{keV}$ with a rate of 62.2 events/(tonne $\times$ year), with the tritium mostly contributing to improving the fit in the bins from $4-7 \mathrm{keV}$.

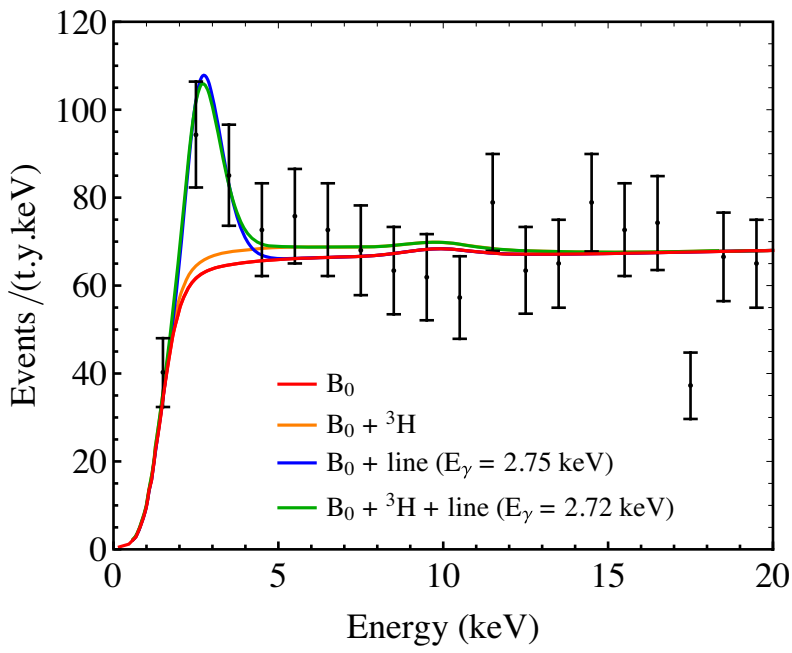

FIG. 1. The best-fit line signal model with (green) and without (blue) the inclusion of a tritium component compared to the background-only event rate (red) and the background-plustritium event rate (orange). 
With the tritium inclusion, the fit becomes $\chi^{2} /$ d.o.f $=0.43$, with $\Delta \chi^{2}=11.7$, compared to the background-only rate. Thus, a prominent line feature is still a significant component of the excess even when an unconstrained tritium component is added. Note that the best-fit energy we obtain is higher than the best fit obtained by XENON1T's bosonic dark matter fit $(E=2.3 \mathrm{keV})$. This is due to the bin width of the provided data. But the best-fit point obtained by the XENON1T collaboration only differs from the best-fit point of this analysis by half of a bin width. In our binned analysis, $\delta=2.3 \mathrm{keV}$ is only disfavored by $\Delta \chi^{2} \sim 1$, so our analysis is consistent with that of XENON1T. Nothing substantial in the model changes if one chooses $\delta=2.3 \mathrm{keV}$, and we see from Fig. 2 that this point is strongly preferred to background.

For comparison with the best-fit point, we evaluate the $\Delta \chi^{2}$ for line energies in the range $E_{\gamma}=1-5 \mathrm{keV}$, where the $\Delta \chi^{2}$ is minimized by finding the best-fit rate and tritium contribution at each energy. The results of this scan are shown in Fig. 2. We find that line signals in the entire range provide a better fit to the data than the background-only model. Before attempting to explain this excess in terms of BSM physics, we stress that the interpretation of the excess as a monoenergetic line stands on its own and could have a standard model (SM) origin. For example, this line is very close to the $\mathrm{x}$-ray line produced when ${ }^{37} \mathrm{Ar}$ decays via $\mathrm{K}$-shell electron capture to ${ }^{37} \mathrm{Cl}$, which can then relax to its ground state by emitting a $2.8 \mathrm{keV}$ photon [45]. With a halflife of 35 days, ${ }^{37} \mathrm{Ar}$ would need to be continuously

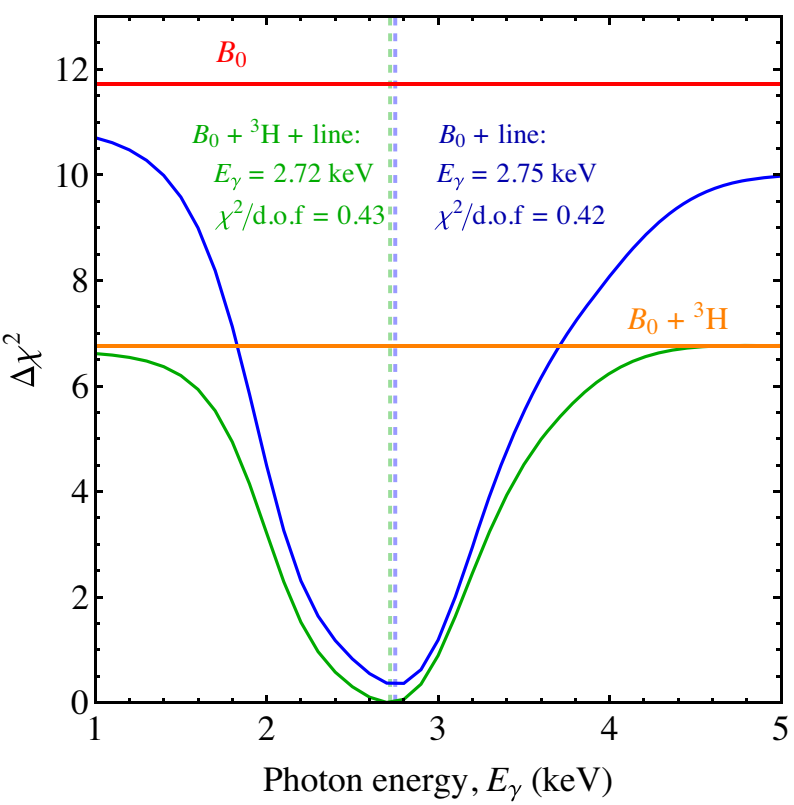

FIG. 2. The $\Delta \chi^{2}$ as a function of the photon energy computed based on the 14 lowest-energy bins for luminous dark matter with (green) and without (blue) the inclusion of a tritium component. For comparison, the background-only $\Delta \chi^{2}$ is also given with (orange) and without (red) the tritium component. introduced throughout the data-taking period, as no time dependence of the rate was found [1]. Without a steady source of ${ }^{37} \mathrm{Ar}$, this explanation of the excess is strongly disfavored.

In the context of LDM, a photon line can provide a viable explanation of the excess so long as a mass splitting of $\sim 2-3 \mathrm{keV}$ is kinematically accessible $\left(m_{\chi} \gtrsim 1 \mathrm{GeV}\right)$, the multiscatter veto is evaded, and constraints from previous low-threshold analyses are not violated. This includes the XENON1T ionization only (S2 only) analysis [46], which constrains both nuclear and electronic recoils, and the standard S1-S2 analysis that constrained nuclear recoils [47]. The nuclear recoil rate for LDM upscatter is given by

$$
\frac{d R}{d E_{R}}=\frac{\rho_{\chi}}{2 m_{\chi} \mu_{\chi N}} \sigma_{\mathrm{SI}} A^{2} F^{2}\left(E_{R}\right) \int_{v>v_{\min }} \frac{f(v)}{v} d v,
$$

where $\rho_{\chi}=0.3 \mathrm{GeV} / \mathrm{cm}^{3}$ is the local dark matter density, $\mu_{\chi N}$ is the dark matter (DM) nucleon reduced mass, $\sigma_{\mathrm{SI}}$ is the spin-independent LDM nucleon cross section, $A$ is the atomic number of the target (we are assuming identical couplings to neutrons and protons), $F^{2}\left(E_{R}\right)$ is the nuclear form factor (taken to be of the Helm form [48]), and $f(v)$ is the velocity distribution (taken to be Maxwellian, with a velocity dispersion of $v_{0}=220 \mathrm{~km} / \mathrm{s}$ and cutoff at $v_{\text {esc }}=544 \mathrm{~km} / \mathrm{s}$ ). The kinematics of inelastic scattering require that the incoming DM particle have a minimum velocity given by

$$
v_{\min }=\frac{E_{R} m_{T}+\delta \mu_{\chi T}}{\sqrt{2 E_{R} m_{T}} \mu_{\chi T}}
$$

where $m_{T}$ is the mass of the target nucleus and $\mu_{\chi T}$ the reduced mass of the $\chi$ and target nucleus.

To check for consistency with previous XENON1T data, we perform a single bin analysis where the total upscattering rate is required to be below the total number of observed events in the signal regions of the two nuclear recoil analyses [46,49]. For simplicity, we perform this analysis for the scenario with LDM only and no tritium. Additionally, the lack of observation of the $\sim 2-3 \mathrm{keV}$ line in the S2-only electron recoil also constrains the cross section. To compute the upper limit, we require that the total number of events in the upper two bins of the S2-only analysis in a 22 tonne-day exposure (19 events). These upper bounds are displayed in Fig. 3 along with the cross section required to explain the excess [i.e., producing a total rate of 69.8 events/(tonne $\times$ year)]. This cross section is given as a range, where the upper limit of the range assumes a worst-case loss of efficiency due to the multiscatter veto. Note that the loss of efficiency will also affect the constraints we have calculated. A full accounting of the effect of the multiscatter veto will require a detailed detector simulation, which is beyond the scope of the present work. 


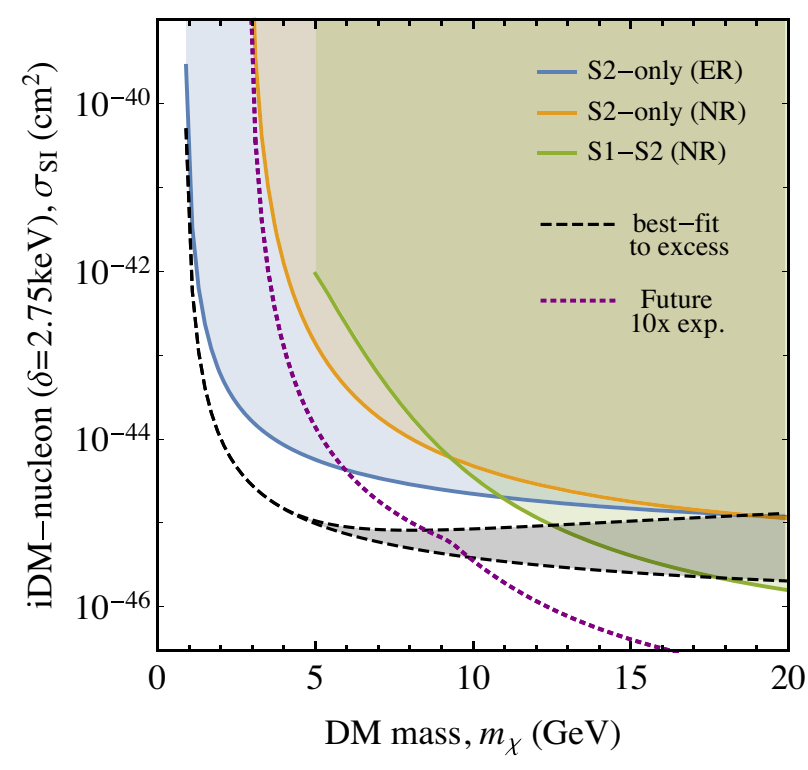

FIG. 3. Constraints on the spin-independent inelastic dark matter (iDM) nucleon cross section for $\delta=2.75 \mathrm{keV}$ derived from other XENON1T analyses [46,49]. Constraints from S2-only data are given for both electron (blue) and nuclear recoils (orange), while the S1-S2 results are only used to constrain nuclear recoils (green). The dashed black denotes the approximate cross section required to explain the excess with a $2.75 \mathrm{keV}$ mass splitting. The purple dotted curve denotes the future limits that could be placed directly on the NewtonRaphson signal with 10 times the exposure.

We find that the LDM scenario is viable for a wide range of DM masses, from $\sim 15-17 \mathrm{GeV}$ down to the kinematic cutoff of $1 \mathrm{GeV}$ where the required cross section is on the cusp of the S2-only entity relationship bound. In the near future XENONnT $[50,51]$ will begin operating. With three times the fiducial mass of XENON1T, it will be able to collect 10 times the exposure of XENON1T in a few years. Assuming a commensurate reduction in the background rate, such an exposure will directly probe the nuclear recoils of this LDM scenario down to $10 \mathrm{GeV}$ in dark matter mass.

Future prospects. - We briefly discuss some avenues for probing this scenario with data from future experiments.

Direct detection spectrum: Upcoming direct detection experiments should be able to distinguish between this scenario and other BSM scenarios and possible tritium backgrounds. In particular, as energy resolution improves, the peak arising from LDM will become increasingly sharp and therefore more easily distinguishable from other possibilities.

Multichannel direct detection signal: Interestingly, because $m_{\chi}$ can be as large as $15 \mathrm{GeV}$, future xenon-based direct detection experiments could potentially see a nuclear recoil signal. If the tail of the nuclear recoil spectrum from inelastic scattering is above threshold, future experiments such as LZ [52], XENONnT [50,51], or PandaX-4T [53] may see both nuclear recoils and the decay signal, which would be a powerful cross-check. In particular, this signal could also help distinguish this scenario from that of bosonic dark matter absorption, which also yields a monoenergetic peak. Specific model details would naturally arise when considering future signals. For example, some models produce lifetimes that would preclude observation of prompt decays within the detector, so one would see nuclear recoils and decay photons, but they would not be from the same event (see, for example, [54] for a recent discussion).

Diurnal modulation: If the decay length is of an order the length of the overburden $\left[\mathcal{O}\left(10^{3}\right) \mathrm{m}\right]$ or greater, then more events will be observed when the dark matter wind passes through the Earth (yielding a larger volume for scattering), while fewer events will be observed if the dark matter wind comes from above the detector. This diurnal modulation was discussed in the context of LDM in [24].

Collider production: $\chi$ or $\chi^{\prime}$ can be produced at beam experiments, yielding either photon or missing energy signatures. For example, the $p p \rightarrow \chi \chi^{\prime} j$ process will yield a monojet and missing energy signal $[55,56]$ if the $\chi^{\prime}$ lifetime is sufficiently large. For a short lifetime (decay within the detector), it will produce monophoton final state [57,58]. However, in order to be observed at the LHC, the boosting of the $\chi^{\prime}$ would have to be substantial, resulting in a $\sigma_{\chi p}$ cross section required by the fit that would be suppressed and mostly out of reach for the LHC.

Beam-dump and fixed-target experiments: $\chi^{\prime}$ can be produced in beam-dump and fixed-target experiments, and if it is long-lived, then an energetic photon spectrum could be seen at FASER [59-62], SHiP [63,64], SeaQuest $[65,66]$, or other displaced detectors. The production cross section, however, will be dependent on the model dependent details of the interaction between dark matter and the standard model.

Summary.-XENON1T has recently reported an interesting unexplained excess of electron recoil events, with typical energies of a few $\mathrm{keV}$. Although this excess can potentially be explained by a tritium background, there has naturally been interest in BSM explanations of this signal. We have shown that this signal can be produced by a species of luminous dark matter with mass in the $\sim 1-15 \mathrm{GeV}$ range, with a mass splitting between the heavy and light states of $2.75 \mathrm{keV}$. If the dark matter scatters inelastically with nuclei in the detector or the surrounding rock, then the heavier state can decay back to the light state within the detector, emitting one or more photons with an energy of $\sim 2.75 \mathrm{keV}$. Including the effects of the energy resolution, this model is a good fit to the data: $\chi^{2} /$ d.o.f $=0.42$, with $\Delta \chi^{2}=11.4$, compared to the background-only case. When we include a tritium contribution, the best-fit line value shifts down to $2.72 \mathrm{keV}$ and the fit becomes $\chi^{2} /$ d.o.f. $=0.43$, with $\Delta \chi^{2}=11.7$, compared to the background-only model. This is a very general framework; the fit to the data depends primarily on the mass 
splitting and the decay channel (to photons) but has very little specific model dependence on the microphysics.

This scenario can be probed with future data from direct detection experiments, which can be used to distinguish this scenario from other BSM scenarios, as well as from the tritium background. As an example, the LDM structure allows for a possible distinctive diurnal feature or multichannel detection, etc., which can be searched for in future experiments. In addition, collider, beam-dump, and fixedtarget experiments can also provide interesting signals, but these are much more dependent on the details of dark matter interactions with the standard model.

We are grateful for helpful discussions with Rafael Lang and Cara Giovanetti. N. F. B. and J. L. N. were supported in part by the Australian Research Council. J. B. D. acknowledges support from the National Science Foundation under Grant No. NSF PHY-1820801. B. D. and S. G. acknowledge support from DOE Grant No. DE-SC0010813. The work of J. K. is supported in part by DOE Grant No. DE-SC0010504.

*Corresponding author.

n.bell@unimelb.edu.au

${ }^{\dagger}$ Corresponding author.

jbdent@shsu.edu

Corresponding author.

ghosh@tamu.edu

${ }^{\S}$ Corresponding author.

dutta@physics.tamu.edu

"Corresponding author.

jkumar@hawaii.edu

Corresponding author.

jayden.newstead@unimelb.edu.au

[1] E. Aprile et al. (XENON Collaboration), arXiv:2006.09721.

[2] N. F. Bell, M. Gorchtein, M. J. Ramsey-Musolf, P. Vogel, and P. Wang, Phys. Lett. B 642, 377 (2006).

[3] N. F. Bell, V. Cirigliano, M. J. Ramsey-Musolf, P. Vogel, and M. B. Wise, Phys. Rev. Lett. 95, 151802 (2005).

[4] M. Agostini et al. (Borexino Collaboration), Phys. Rev. D 96, 091103 (2017).

[5] L. Di Luzio, M. Fedele, M. Giannotti, F. Mescia, and E. Nardi, arXiv:2006.12487 [Phys. Rev. Lett. (to be published)].

[6] F. Takahashi, M. Yamada, and W. Yin, this issue, Phys. Rev. Lett. 125, 161801 (2020).

[7] A. Bally, S. Jana, and A. Trautner, preceding Letter, Phys. Rev. Lett. 125, 161802 (2020).

[8] K. Kannike, M. Raidal, H. Veerme, A. Strumia, and D. Teresi, arXiv:2006.10735.

[9] G. Alonso-lvarez, F. Ertas, J. Jaeckel, F. Kahlhoefer, and L. Thormaehlen, arXiv:2006.11243.

[10] d. Amaral, D. W. Praia, D. G. Cerdeno, P. Foldenauer, and E. Reid, arXiv:2006.11225.

[11] B. Fornal, P. Sandick, J. Shu, M. Su, and Y. Zhao, following Letter, Phys. Rev. Lett. 125, 161804 (2020).
[12] C. Boehm, D. G. Cerdeno, M. Fairbairn, P. A. Machado, and A. C. Vincent, arXiv:2006.11250.

[13] J. Smirnov and J. F. Beacom, arXiv:2002.04038 [Phys. Rev. Lett. (to be published)].

[14] K. Harigaya, Y. Nakai, and M. Suzuki, Phys. Lett. B 809, 135729 (2020).

[15] M. Du, J. Liang, Z. Liu, V. Q. Tran, and Y. Xue, arXiv:2006.11949.

[16] G. Choi, M. Suzuki, and T. T. Yanagida,arXiv:2006.12348.

[17] Y. Chen, J. Shu, X. Xue, G. Yuan, and Q. Yuan, arXiv:2006.12447.

[18] D. A. Sierra, V. De Romeri, L. Flores, and D. Papoulias, Phys. Lett. B 809, 135681 (2020).

[19] G. Paz, A. A. Petrov, M. Tammaro, and J. Zupan, arXiv:2006.12462.

[20] J. Buch, M. A. Buen-Abad, J. Fan, and J. S. C. Leung, arXiv:2006.12488.

[21] J. Bramante and N. Song, this issue, Phys. Rev. Lett. 125, 161805 (2020).

[22] B. Feldstein, P. W. Graham, and S. Rajendran, Phys. Rev. D 82, 075019 (2010).

[23] M. Pospelov, N. Weiner, and I. Yavin, Phys. Rev. D 89, 055008 (2014).

[24] J. Eby, P. J. Fox, R. Harnik, and G. D. Kribs, J. High Energy Phys. 09 (2019) 115.

[25] D. Smith and N. Weiner, Phys. Rev. D 64, 043502 (2001).

[26] D. Tucker-Smith and N. Weiner, Phys. Rev. D 72, 063509 (2005).

[27] D. P. Finkbeiner and N. Weiner, Phys. Rev. D 76, 083519 (2007).

[28] C. Arina and N. Fornengo, J. High Energy Phys. 11 (2007) 029.

[29] S. Chang, G. D. Kribs, D. Tucker-Smith, and N. Weiner, Phys. Rev. D 79, 043513 (2009).

[30] Y. Cui, D. E. Morrissey, D. Poland, and L. Randall, J. High Energy Phys. 05 (2009) 076.

[31] P. J. Fox, G. D. Kribs, and T. M. P. Tait, Phys. Rev. D 83, 034007 (2011).

[32] T. Lin and D. P. Finkbeiner, Phys. Rev. D 83, 083510 (2011).

[33] A. De Simone, V. Sanz, and H. P. Sato, Phys. Rev. Lett. 105, 121802 (2010).

[34] H. An, P. S. B. Dev, Y. Cai, and R. N. Mohapatra, Phys. Rev. Lett. 108, 081806 (2012).

[35] D. P. Finkbeiner and N. Weiner, Phys. Rev. D 94, 083002 (2016).

[36] K. R. Dienes, J. Kumar, B. Thomas, and D. Yaylali, Phys. Rev. Lett. 114, 051301 (2015).

[37] G. Barello, S. Chang, and C. A. Newby, Phys. Rev. D 90, 094027 (2014).

[38] J. Bramante, P. J. Fox, G. D. Kribs, and A. Martin, Phys. Rev. D 94, 115026 (2016).

[39] N. F. Bell, G. Busoni, and S. Robles, J. Cosmol. Astropart. Phys. 09 (2018) 018.

[40] J. R. Jordan, Y. Kahn, G. Krnjaic, M. Moschella, and J. Spitz, Phys. Rev. D 98, 075020 (2018).

[41] A. Migdal, J. Phys. Acad. Sci. USSR 4, 449 (1941).

[42] M. Ibe, W. Nakano, Y. Shoji, and K. Suzuki, J. High Energy Phys. 03 (2018) 194.

[43] M. J. Dolan, F. Kahlhoefer, and C. McCabe, Phys. Rev. Lett. 121, 101801 (2018). 
[44] N. F. Bell, J. B. Dent, J. L. Newstead, S. Sabharwal, and T. J. Weiler, Phys. Rev. D 101, 015012 (2020).

[45] M. Alfonsi, Understanding neutrino background implications in lxe-tpc dark matter searches using $127 x$ e electron captures (2019), IIDINE.

[46] E. Aprile et al. (XENON Collaboration), Phys. Rev. Lett. 123, 251801 (2019).

[47] E. Aprile et al. (XENON Collaboration), Phys. Rev. Lett. 121, 111302 (2018).

[48] J. Lewin and P. Smith, Astropart. Phys. 6, 87 (1996).

[49] E. Aprile et al. (XENON Collaboration), Phys. Rev. Lett. 123, 241803 (2019).

[50] A. Brown, Status and Results from the XENON Dark Matter Project (2019), EPS-HEP 2019.

[51] E. Shockley, Search for New Physics with Electronic-Recoil Events in XENON1T (2020), LNGS Webinar.

[52] D. Akerib et al. (LZ Collaboration), Nucl. Instrum. Methods Phys. Res., Sect. A 953, 163047 (2020).

[53] H. Zhang et al. (PandaX Collaboration), Sci. China Phys. Mech. Astron. 62, 31011 (2019).

[54] M. Baryakhtar, A. Berlin, H. Liu, and N. Weiner, arXiv:2006.13918.
[55] M. Aaboud et al. (ATLAS Collaboration), J. High Energy Phys. 01 (2018) 126.

[56] V. Khachatryan et al. (CMS Collaboration), Eur. Phys. J. C 75, 235 (2015).

[57] S. Baek and T. Jung, in The First MadAnalysis 5 Workshop on LHC Recasting (2018), pp. 23-26, https://inspirehep.net/ literature/1676808.

[58] A. M. Sirunyan et al. (CMS Collaboration), J. High Energy Phys. 10 (2017) 073.

[59] J. L. Feng, I. Galon, F. Kling, and S. Trojanowski, Phys. Rev. D 97, 035001 (2018).

[60] A. Ariga et al. (FASER Collaboration), arXiv:1811.10243.

[61] A. Ariga et al. (FASER Collaboration), arXiv:1812.09139.

[62] A. Ariga, T. Ariga, J. Boyd, F. Cadoux, D. W. Casper et al. (FASER Collaboration), Phys. Rev. D 99, 095011 (2019).

[63] M. Anelli et al. (SHiP Collaboration), arXiv:1504.04956.

[64] S. Alekhin et al., Rep. Prog. Phys. 79, 124201 (2016).

[65] C. Aidala et al. (SeaQuest Collaboration), Nucl. Instrum. Methods Phys. Res., Sect. A 930, 49 (2019).

[66] A. Berlin, S. Gori, P. Schuster, and N. Toro, Phys. Rev. D 98, 035011 (2018). 